\title{
Review on the Development of Physical Agricultural Technology in China
}

\author{
Zhang $\mathrm{Fu}^{1}$, Fu Sanling ${ }^{2}$ \\ ${ }^{1}$ (College of Vehicle \&Motive Power Engineering, Henan University of Science and Technology \\ Luoyang, P R China) \\ ${ }_{2}^{2}$ (College of Physics and Engineering, Henan University of Science and Technology \\ Luoyang, P R China)
}

\begin{abstract}
With the development of agriculture, modern physical agricultural technology have made a lot of research achievements, and more and more experts and farmers received more attention and recognition on physical agricultural technology. The characteristics of the practical physical agricultural technology was analyzed, the common problems and the development of the physical agriculture were discussed. The development thought of the physical agricultural technology which strengthen basic theory research of modern physical agricultural engineering technology involved in the agriculture science, environmental science, biologic science and physical science, and increase the demonstration to popularize strength of a national modern physical agricultural technology demonstration project, set up science and technology demonstration base, park, and guide peasants to know and adopt the modern physical agricultural engineering technology.
\end{abstract}

Keywords - Review, physical agriculture, technology, equipment

\section{INTRODUCTION}

M odern agriculture is the latest stage of agricultural development which included marked characteristics of realizing agricultural mechanization, the optimization of the production structure and greatly improving agricultural productivity. At present, chemical fertilizer and chemical pesticide, as a representative of the chemical agriculture technology brings the agricultural revolution, which has greatly enhanced the crop production. But, the crop yields excessively depend on the chemical fertilizers and pesticides for human beings, which made the soil degradation, the aggravating pollution, the environmental degradation and the ecological destruction get more and more serious within the scope of the world, and so the research of the agricultural sustainable development gradually made to the agenda date $[1,2]$.

The development of ecological agriculture is the important action to realize the agricultural sustainable development. The world agriculture is shifting from the traditional chemical agriculture to the ecological agriculture, and the main way of the transformation is biological engineering and physical agriculture. Physical agriculture as modern agriculture science and technology research new areas is dependent on the modern physics foundation and the development of agricultural practical technology $[3,4]$.

The physical principle with the electricity, the magnetics, the acoustics, the optics and the thermodynamics serves as the technology base of the modern physical agriculture. The agricultural products was used by the specific physical technology to improve the agricultural production environment, reduce chemical fertilizers and pesticides, change agricultural production means, improve agricultural production quantity and quality, increase the ability of the ability of resistant to diseases and insects [5,6]. In the paper, the characteristics of the practical physical agricultural technology was analyzed, the common problems and the development of the physical agriculture were discussed.

\section{THE DEVELOPMENT SITUATION OF THE MAJOR PHYSICAL AGRICULTURAL TECHNOLOGY}

With the development of agriculture, modern physical agricultural technology have made a lot of research achievements, and more and more experts and farmers received more attention and recognition, especially the physical agricultural technology get good applications in Tianjin city, Dalian city, Qingdao city of China, and the government department also encourage and support modern physical agriculture. The modern physical agricultural equipment can be indexed by the purchase subsidies directory of local agricultural machinery in Tianjin City[7,8]. At present, more and more scientific research institutions and the enterprises research and develop physical agricultural technology in China.

\subsection{Sound Waves Promotion Technology}

Sound waves promotion technology of the crops were researched based on the characteristic of sound, and the sound waves with specific frequency was imposed on the crops by the loudspeaker box, the resonance phenomenon will produce when the frequency of sound waves and the inherent physiological system frequency wave of the crops is consistent, which can improve the movement velocity of the current of the crops in living cells, promote the process of the absorption, the transmission and the transformation with all sorts of nutrition elements, enhance the crops photosynthesis, 
create the synthetic organic matter with protein and sugar, and make the crops show strong growth trends and increase the effects of the quality and the paedogenesis of the crops [9]. In addition, the sound box of the resonant wave can still improve crops growth ability in vigorous station, so as to keep the cell oxidation of the higher level, and breathing can also provide energy and intermediate products which are helpful for the crops form some isolation to prevent disease expanding.

The crops sound wave generator was researched by Dalian agricultural mechanization institute in China. The figure of the crops sound wave generator was showed as Figure 1. The crops was influenced by the special sound waves frequency of the crops sound wave generator based on sound frequency of the crops and the relations with the plant biological acoustic characteristics and the environmental factors.

\subsection{The Seed Treatment Technology}

People began to use various physical means to improve crop seeds quality in order to achieve the purpose of the yields from the late $1980 \mathrm{~s}$.

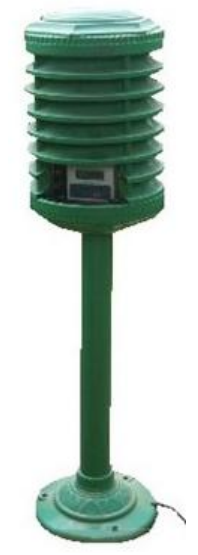

Figure 1 The sound wave generator with SZ-C type

The test shows that the pesticide and the fertilizer will be reduce or reduce as little as possible, the quality and the seed germination rate of the crops will be obviously increasing, the healthy and uniform advantage of the seedling was obvious after the seed processing by the specific physical processing technology, which will preferably solve the phenomenon of three generations seedling and the disease resistance level of the crops, and the ability of the resisting natural disasters and the acclimatization were effectively increasing[10]. The figure of the magnetic field seed processor by Zhengjiang modern agricultural equipment Co. LTD in China was showed as Figure 2.

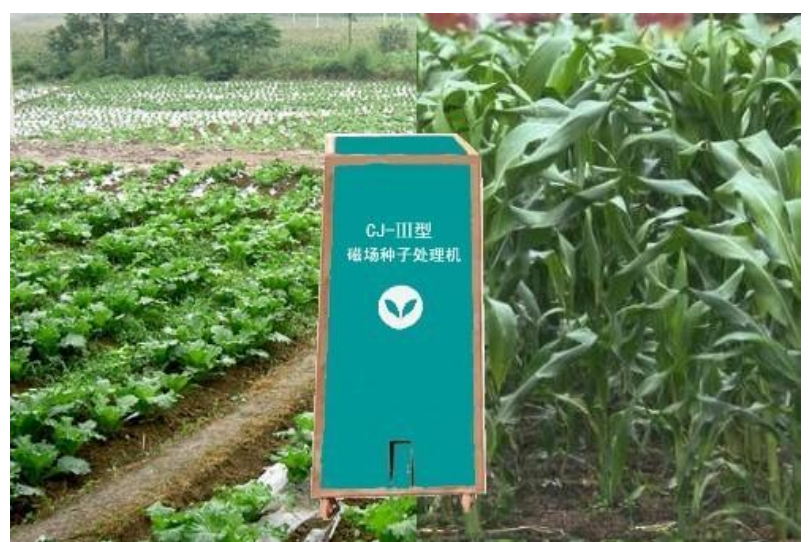

Figure 2 The figure of the magnetic field seed processor with $\mathrm{CJ}-$ III type

\subsection{Filling Light Technology in the Greenhouse}

All over now out-of-season vegetable, melon, fruits or flowers were widely produced by the greenhouse, but general illumination in a greenhouse is inadequate because of the greenhouse structure, the time of the roller shutters and the effect of the dust and the dew on the plastic films. The filling light technology or technology was adopted to improve the yields and the quality of the crops in the greenhouse. The figure of the sodium lamp to fill light in the greenhouse by Jiangsu Huanyu Greenhouse Equipment Co. Ltd in China was showed as Figure 3.

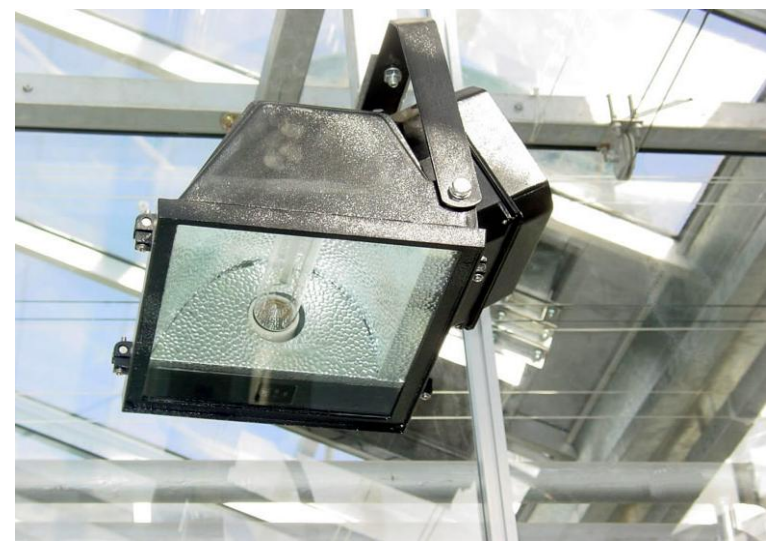

Figure 3 The figure of the sodium lamp to fill light in the greenhouse

\subsection{The Bio-stimulation Technology of the Electric field in the Space}

A great number of charged ions were produced through the space direct electric field, and the fog, the dust or other suspended solids were charged to directional removal movement by the charged ions, which can quickly adsorb on the ground, the wall, the surface of the crops, the most of the pathogenic microbes attached the fog or the dust will be killed on the directional removal process in the high-energy charged particles at the same time [11]. The rising fog and the dust in the sky were suppressed by the electric field in the space, and the flow of air transmission diseases channels was cut off, it can make the production environment continue to 
keep less toxic bacteria, and the technology has applied in the greenhouse, the corral housing and the mushroom.

\subsection{Electronic Insecticidal Technology}

The pests were annihilated used by the specific spectrum light source and the annihilating equipment based on the phobophototaxis character of the insects by electronic insecticidal technology. Electronic insecticidal lamp included two kinds with the frequency vibration type and the mutual inductance type, and the power supply patterns with the alternating current (AC), the direct current (DC), the solar battery and other forms was adopted.

At present, more than ten enterprises in China had been producing this equipment, and the application field of the technology is different with the changes of the product type, the specification and so on[12]. The electronic insecticidal lamp was applied to the open air, the mushroom and the greenhouse based on the small volume, convenient suspension, and some kinds of electronic insecticidal lamp was suitable for in the plains, the mountains and the hills and the different terrains of the outdoor. Electronic insecticidal technology and equipment in China has greater range promotion use, equipment production has been achieved larger batches $[13,14]$. The figure of the ultraviolet lamp by the Shenshi sunshine technology Co. LTD of changping district of Beijing in China was showed as Figure 4.
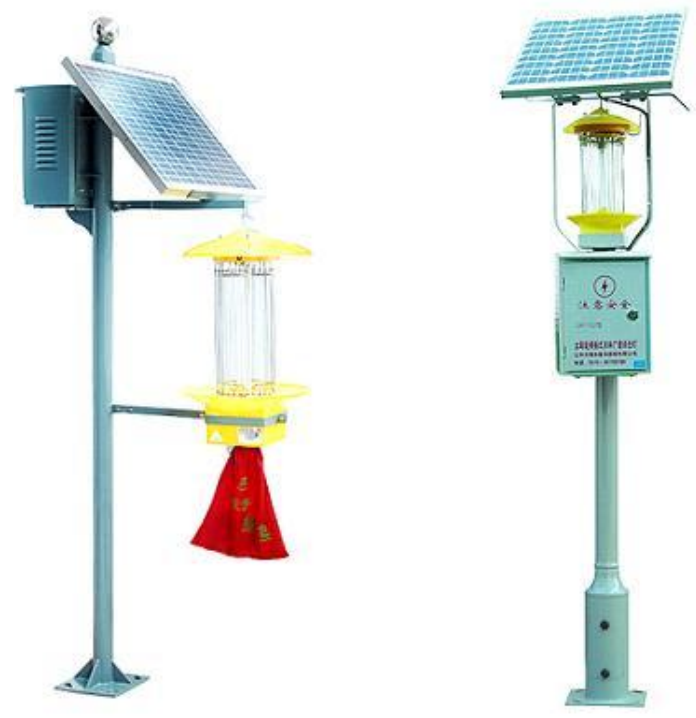

Figure4 The figure of the ultraviolet lamp

\subsection{Water Treatment Technology}

Good water quality can effectively promote the animal and plant growth ability. Lishui Agricultural Sciences Research Institute of Zhejiang province in China researched the electric water generator and the intelligent electric field system, and water under the action of the electric field will produce two kinds waters included the acid water and the alkali water, which used in the sterilization during crops growth situation and the cleaning process of the agricultural products. The electric function water will restore or oxidize to the ordinary water in the role of the process, which will realize the function of the pollution-free and the recycling of the water[15]. The equipment or technology in Japan and other developed countries received universal applications, and the technology in China started late, but also the good application effects was created, and the basic technology was developing to serial production[16,17]. In addition, the magnetized water device was produced in China enterprises, and the hydrogen bond of the water molecules was instantaneously broken through electromagnetic field to improve the water activity [18]. The crops used by the magnetized water included many prominent characteristics with the safe, the non-pollution and the creating yield and quality of agricultural products. The figure of the electric water generator by Lishui Agricultural Sciences Research Institute of Zhejiang province in China was showed as Figure 5.

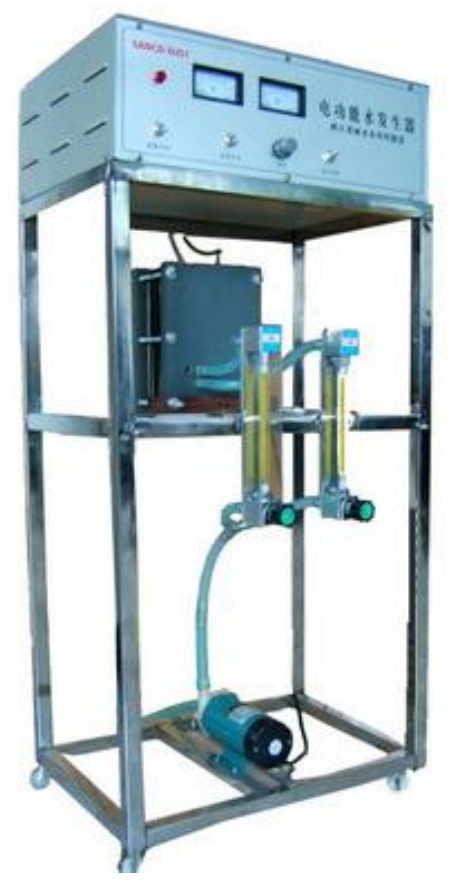

Figure 5 The figure of the electric water generator

\section{The MaIN Problems IN THE Modern Physical Agricultural Technology}

\subsection{The Low Theory Research Level}

At present, the modern physical agricultural technology has made the good application effects, but the overall level of the technology development is not high, part of the technology still not mature, the increasing efficiency of the effects are not obtain the strict verification on theory level, which will lead to instability application effect. The main reason is that the influence of many factors of agricultural production and theory and the study itself has a lot of difficulty $[3,9]$. 


\subsection{Lack of Technology Integration Research}

The modern physical agricultural technology research tends to a single technology equipment development, but comprehensive application effect is not apparent, which can obstruct the application of the physical agriculture [3]. The technology effectively integrated to meet the needs of the whole process of agricultural production, which will become the hot spot of the modern physical agricultural research.

\subsection{Small Application Ranges}

Now the development and production of the modern physical agricultural technology enterprise is not much in China, the development of the technology is still in the initial stage, and development production of equipment can not completely meet the need of agricultural production[3,9], for example, most equipment used in the crops, but less used for animal husbandry, fishery and other areas of equipment.

\section{CONCLusions}

Strengthen basic theory research of modern physical agricultural engineering technology involved in the agriculture science, environmental science, biologic science and physical science included the electricity, the magnetics, the acoustics, the optics, the thermodynamics, etc. The relevant universities and research institutes should strengthen basic theory research and strengthen the cooperation between the production, the study and the research, which will raise the overall physical agricultural technology and equipment development production level, and promote technical achievements transformation into direct productivity as soon as possible.

Increase the demonstration to popularize strength of a national modern physical agricultural technology demonstration project, set up science and technology demonstration base, park, and guide peasants to know and adopt the modern physical agricultural engineering technology. At the same time, the government should increase the modern physics of agricultural transformation of scientific and technological achievements, to support the transformation of scientific and technological achievements, strengthen the propaganda, and improve the technology of conversion speed.

\section{ACKNOWLEDGEMENTS}

This work was supported by Science and Technology Project of Henan province (Grant No.112102310476), Natural Science Foundation of Henan Educational Committee (Grant No. 2009B210006), Scientific Research Foundation for Ph. Doctor and the Experiment Technology Foundation of 20072008 Academic Year (SY0910054) of Henan University of Science and Technology.

\section{REFERENCES}

[1] $\mathrm{Hu}$ Wei, The modern physical agricultural engineering technology Conspectus, Tianjin Science and Technology press, 2011.

[2] Hao Shuiyuan, Developing Physical Agriculture for
Ecological Agriculture, Journal of Hetao University, 2008, 5, 27-34.

[3] Hu Wei, Song Ying, The application and development of modern physical agricultural engineering technology in our country, Agricultural Technology \& Equipment, 2010, 15-18.

[4] Bai Yaxiang, Hu Yucai and Chi Jianwei, Application Progress of Phycical Techniques in Agriculture, Journal of Shenyang Agricultural University, 2003, 34, 232-235.

[5] Song Ying and Hu Wei, Research on Modern Physical Agricultural Engineering Technology System, Agricultural Engineering, 2011, 1, 8-11.

[6] Song Ying and Hu Wei, The development situation of the modern physical agricultural equipment, Agriculture Machinery Technology Extension, 2010, 51-53.

[7] Wang Yanhong, New Developments of Agricultural Engineering Discipline, Agricultural Engineering, 2011, 1, $114-115$.

[8] Fu Sanling, Zhang Fu, Li Jiangchang, Wang Baozhu and Yang Xin, Application and Trend about Several Physics Technology in Agricultural Fields, Journal of Agricultural Mechanization Research, 2006, 36-38.

[9] Chen Yong and Wang Qingying, The equipment and technology of the physical agriculture, Agricultural Technology \& Equipment, 2010, 65-67.

[10] Hu Lianglong, Tian Lijia, Hu Zhichao, Wang Haiou and Wu Feng, Application of Physical Agriculture Techniques in Cleaned Seeds Treatments, Journal of Anhui Agricultural Sciences, 2007, 35, 3778-3779.

[11] Huang Xiantun, The physical interpretation of mechanism in high voltage electrostatic field and its application in agriculture, Guangdong Agricultural Sciences, 2010, 189-191.

[12] Hu Fanglin, On the Physical Agricultural Technology Application and Prospects in China, Journal of Agricultural Mechanization Research, 2008, 250-252.

[13] Liu Mingwei. Applied Research of Practical Techniques of Physical Agriculture, Agricultural Science \& Technology and Equipment, 2010, 60-61.

[14] Xu Jie, The Relationship between Physical Techniques and Agricultural Modernization, Inner Mongolia Agricultural Science and Technology, 2007, 85-87.

[15] Xu Zhiying, Chen Bo, Wei Zhen, Overview on Physical Agriculture Technology, Agricultural Science \& Technology and Equipment, 2011, 103-104.

[16] Zhu Jie, Guo Lianhong, and Wang Guodong., A discussion of physical agriculture and agricultural physics and their roles on agricultural modernization in China, Journal of Agricultural Sciences, 2008, 29, 64-67.

[17] Zhu Jie, Guo Lian-hong and Wang Guo-dong, Applications of physical agricultural techniques in fundamental agricultural science and engineering, College Physics,2008, 46-49.

[18] Zhang Hua, The application of physical agricultural technology, Modern Agricultural Equipments, 2009, 54-55. 